\title{
Personalia
}

Herr PD Dr. med. Martin Freesmeyer ist im Januar 2021 zum außerplanmäßigen Professor der Friedrich-Schiller-Universität Jena für das Fach Nuklearmedizin ernannt worden.

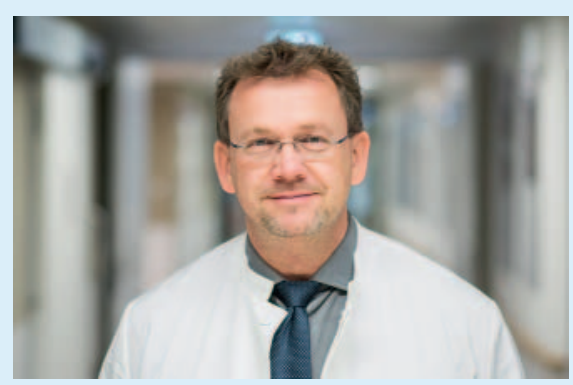

Professor Martin Freesmeyer.

Quelle: Schroll/UKJ.

Nach seinem Medizinstudium in Jena war Herr Professor Freesmeyer ab dem Jahr 1998 am Institut für Diagnostische und Interventionelle Radiologie des Uniklinikums Jena tätig und erlangte im Jahr 2003 die Anerkennung zum Facharzt für Diagnostische Radiologie. Ab 2004 absolvierte er eine nuklearmedizinische Weiterbildung am Universitätsklinikum Halle und ist seit 2007 Facharzt für Nuklearmedizin.

Seit dem Jahr 2007 ist Herr Professor Freesmeyer als Chefarzt der Klinik für Nuklearmedizin des Universitätsklinikums Jena tätig, wobei er unabhängig und selbstständig Forschung, Lehre und Krankenversorgung gestaltet. Im Jahr 2014 habilitierte er sich an der Medizinischen Fakultät der Friedrich-Schiller-Universität Jena. Seine Habilitationsschrift verfasste er zum Thema „Entwicklungen und Untersuchungen zur Bildgebung der Schilddrüse: 124lod-PET/CT, 3D-Ultraschall und nuklearmedizinisch-sonographische Bildfusion“.

Im Jahr 2017 erfolgte die erfolgreiche Inbetriebnahme eines Klinikneubaus in Jena mit einem modernen nuklearmedizinischen GMPLabor und einer neuen Therapiestation. Aus der wissenschaftlichen Tätigkeit der letzten Jahre sind zahlreiche Publikationen u. a. im Journal of Nuclear Medicine und in der Zeitschrift Radiology sowie 3 Patentanmeldungen hervorgegangen. Herr Professor Freesmeyer wurde durch die Studierenden der Medizinischen Fakultät 3-mal für die Qualität und das Engagement in der Lehre ausgezeichnet und zuletzt im Jahr 2020 mit dem Janus-Cornarius-Lehrpreis der Fachschaft geehrt.

Im Dezember 2020 ist Herr PD Dr. med. Jan Sommerlath Sohns zum außerplanmäßigen Professor der Medizinischen Hochschule Hannover ernannt worden.

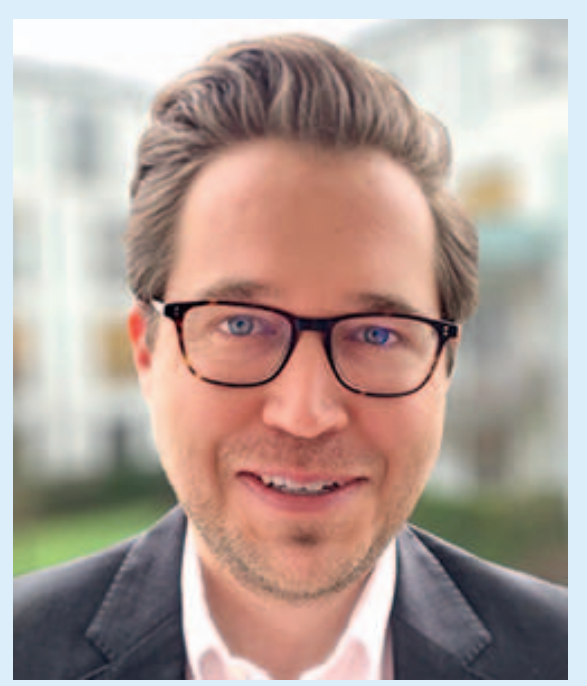

Professor Sommerlath Sohns.

Quelle: Jan Sommerlath Sohns, Köln.

Nach seiner Promotion zum Thema „Funktionelle MRT“ in der Klinik für Neurologie der Universitätsmedizin Göttingen war Herr Professor Sommerlath Sohns ab dem Jahr 2010 in der Radiologie sowie auch Neuroradiologie der Universitätsmedizin Göttingen tätig. Später engagierte er sich als Nachwuchsforscher im Deutschen Zentrum für Herz-Kreislauf-Forschung (DZHK) und arbeitete parallel an einem Forschungspro- jekt zu „Real-Time-MRI“ mit dem MaxPlanck-Institut für Biophysikalische Chemie.

Im Jahr 2015 habilitierte er sich im Fach Radiologie an der Georg-August-Universität Göttingen und wurde im selben Jahr zum Facharzt für Radiologie ernannt. Seine Habilitationsschrift verfasste er zum Thema „Bedeutung anatomischer Pathologien im gesamten Untersuchungsbereich diagnostischer Verfahren am Beispiel der Magnetresonanztomographie und Computertomographie“. Im Rahmen seiner Habilitation hielt er sein Kolloquium zum Thema „Kardiale MRTBildgebung in Echtzeit - Real-Time-MRI“.

2016 wechselte er an die Klinik für Nuklearmedizin der Medizinischen Hochschule Hannover, an der er bis heute auch als Dozent tätig ist. Dort beschäftigte er sich mit der FDG-PET/CT-Entzündungsbildgebung von linksventrikulären Unterstützungssystemen (LVAD).

Im Jahr 2020 wurde Herr Professor Sommerlath Sohns noch zum Facharzt für Nuklearmedizin ernannt.

Die DGN gratuliert Herrn Professor Freesmeyer und Herrn Professor Sommerlath Sohns sehr herzlich.

VERANTWORTLICH FÜR DEN INHALT

\section{Deutsche Gesellschaft für}

Nuklearmedizin e. V. (DGN)

Prof. Dr. Bernd Joachim Krause (Präsident)

Nikolaistr. 29, 37073 Göttingen

E-Mail: office@nuklearmedizin.de

\section{Schriftführer:}

Prof. Dr. Peter Bartenstein

E-Mail: office@nuklearmedizin.de 